\title{
Study of Stromal Femtosecond Laser Ablation for Deep Corneal Cut Optimization
}

\author{
A. Bernard $^{*^{1} *^{3}}$, E. Audouard $*^{2} *^{3}$, G. Thuret $*^{1}$, M. Peoc' $h^{* 1}$, J-M. Dumollard $*^{1}$, J.Granier $*^{3}$, B.Moine $*^{2}$, H. \\ Soder*3 ${ }^{3}$ P. Gain*1 \\ ${ }^{*}$ Université Jean Monnet, Saint-Étienne, Laboratory « Biology, Engineering and Imaging of corneal graft » BiiGC \\ (EA 2421) Faculty of Medicine, 15 rue Ambroise Paré, 42023 Saint Étienne, France \\ ${ }^{* 2}$ Université de Lyon, F-69003, Lyon, France, Université de Saint-Étienne, Laboratoire Hubert Curien (UMR 5516 \\ CNRS), 42000 Saint Étienne, France \\ *3 IMPULSION SAS, Pole Optique Vision, 12, rue B. Lauras, 42000 Saint Etienne, France
}

E-mail: aurelien.bernard@univ-st-etienne.fr

\begin{abstract}
Femtosecond laser (FL) has become a common tool in corneal surgery during the last years. The first clinical application of FL in ophthalmology was in refractive surgery for the cutting of superficial cornea flaps in transparent corneas. Recently, FL applications have been extended to penetrating and anterior or posterior lamellar corneal grafts. However, commercially available FL in ophthalmology have already shown limitations in these new applications, particularly in oedematous cornea (secondary to corneal diseases or occurring during storage by eye banks) that cause light scattering during stromal crossing. In order to improve these cuts, knowledge on the biological material, namely corneal stroma, is essential. Moreover, collagen organization of anterior corneal stroma is more compact compared to the posterior one, but the influence of these structural differences on the cut has never been studied. In this work, we compared ablation rates (ARs) in anterior and posterior human corneal stroma, isolated from the rest of the cornea by mechanical dissection in order to eliminate the scattering effect. ARs were quantified using Second Harmonic Generation microscopy. Results showed significantly higher ARs in posterior corneal stroma $(+23 \pm 12 \%$ $($ mean $\pm \mathrm{SD}) ; \mathrm{P}=0.0038)$. Previously reported limitations to cut the posterior stroma with FL can therefore not be explained by the difference in collagen organization but by the optical scattering during stromal crossing.

DOI:10.2961/jlmn.2012.03.021
\end{abstract}

Keywords: cornea, femtosecond laser, ablation rate, second harmonic generation, stroma, graft, human, eye banks

\section{Introduction}

Femtosecond laser has become a common tool in corneal surgery during the last years, combining high precision ablation and minimal collateral tissue damage $[1,2]$. The first clinical application of femtosecond laser (FL) in ophthalmology was in refractive surgery (LASIK : laser in situ keratomileusis) for the preparation of cornea flaps [3] and had recently been extend to penetrating and lamellar keratoplasty [4], intracorneal ring segments implantation in keratoconus eyes [5] and cataract surgery [6].

However, commercially available FLs in ophthalmology have already shown limitations in some applications, in particular in oedematous cornea (pathologic or stored by eye banks). Experimental and clinical investigations of deep lamellar and penetrating graft in oedematous corneas have often shown bad quality dissections, with the presence of numerous remaining tissue bridges $[7,8]$.

In order to improve dissections in the stroma, knowledge of the dissected biological material and a best ablation process control are essential. The human cornea is a complex tissue made of different layers. The stroma, central layer mainly made of collagen fibrils and water, repre- sent more than $90 \%$ of the whole corneal thickness. Collagen fibril organization and hydration in stroma are not homogeneous. Anterior stroma owns a complex collagen fiber branching patterns, which decrease logarithmically to posterior direction $[9,10]$. Transverse shear moduli decrease in the same way from anterior to posterior stroma [11]. Impact on corneal ablation threshold (AT) of pulse duration $[12,13]$, wavelength [14] and depth [15] has already been studied. These works show a decrease of AT with diminution of pulse duration and wavelength and no significant dependence with depth on AT in the first $200 \mu \mathrm{m}$ of anterior stroma. Moreover, no systematic studies of the ablation rate (AR) have been done on cornea despite the wide use of FL in ophthalmology.

In the this work, we report a systematic study of AR on anterior and posterior stromal surface, using a mechanical microkeratome preparation of tissue samples and imaging by Second Harmonic Generation (SHG) microscopy. 


\section{Experimental set-up}

\subsection{Samples preparation}

Four human corneal grafts unsuitable for transplantation for serological reasons have been prepared with a mechanical microkeratome (Moria, Antony, France). Initially stored in a commercially organoculture medium (CorneaMax, Eurobio, les Ulis, France) corneas were transferred in a deswelling medium (CorneaJet, Eurobio) 24h before the cut. Using microkeratome, a lamellar cut from anterior side has been performed at two different depths: $50 \mu \mathrm{m}$ for anterior stroma study and $350 \mu \mathrm{m}$ for posterior stroma study (see figure 1-A). This preparation was intended to prevent the interference between the incident laser and the other corneal layers. The remaining corneas were stored for $24 \mathrm{~h}$ in deswelling medium until the laser processing.

\subsection{Laser processing}

The laser source was an ultrafast laser system delivering $150 \mathrm{fs}$ pulses $(800 \mathrm{~nm})$ at $5 \mathrm{kHz}$ frequency (Bright, Thales, Paris, France). A $7 \mathrm{~mm}$ diaphragm was used with a $20 \mathrm{X}$ long working distance objective. The resulting waist was $4.8 \pm 1.2 \mu \mathrm{m}$ (measured with a beam profiler).

Grooves were realized in the remaining stroma after microkeratome cut (figure 1-B) by moving the sample according $\mathrm{Y}$ on a XYZ translation stages (Schneeberger, Roggwil, Switzerland), $\mathrm{Z}$ being the laser propagation axis. Different pulses number were obtained by modifying translation stages speed and various fluences/pulse have been achieved by adjusting laser power delivered on the sample with a combination of a half-wave-plate and a polarizer. Focalization on stromal surface was realized by visualizing the sample on a CCD detector combined with a homemade microscope.

During processing, cornea was maintained in an artificial anterior chamber (Baron Precision Instruments, Grand Blanc, Michigan) with $20 \mathrm{mmHg}$ pressure on endothelial side.

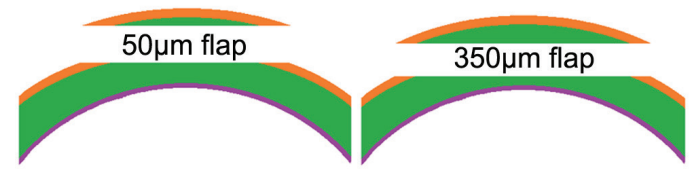

1 - Anterior or posterior stromal surface preparation

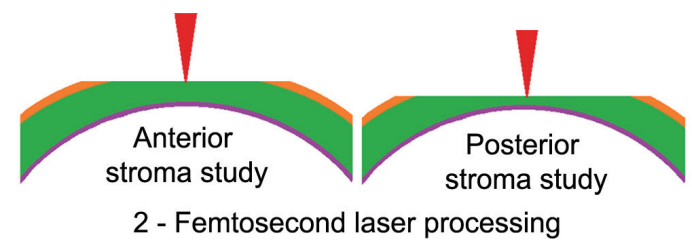

Figure 1: (A) Mechanical microkeratome preparation of corneas in order to study anterior (left) and posterior stroma (right); (B) Processing of grooves in the remaining anterior (left) and posterior stroma (right).

\subsection{SHG imaging}

Immediately after laser processing, corneas were fixed in $4 \%$ paraformaldehyde. During imaging, corneas were maintained on a homemade transparent support, in order to preserve corneal curve and facilitate grooves imaging.

SHG imaging was performed in transmission with a laser scanning inverted microscope (Leica SP2), using a femtosecond laser source (Coherent, Chameleon VI) delivering $140 \mathrm{fs}$ pulses at $840 \mathrm{~nm}$. High numerical aperture water-immersion objective (X40, 0.8NA) was used to achieve lateral and axial resolution of $500 \mathrm{~nm}$ and $1.2 \mu \mathrm{m}$ respectively at $840 \mathrm{~nm}$. Instead of water, an aqueous gel was used as immersion medium.

36 laser-induced grooves were performed, which represent a data set wide enough for statistical analysis. Ablation rates in the anterior and posterior stroma were compared using the complete data set, with a non-parametric test (Wilcoxon signed rank test) with $\mathrm{P}<0.05$ deemed as significant.

\section{Results and discussion}

Figure 2 showed SHG microscopy images of grooves realized in the stroma. On XY sections (A.1 and B.1) background is characterized by striated spatial features, representative of collagen organization. As previously reported [10], anterior stroma (A.1) showed a higher interwoven lamellar organization than posterior stroma (B.1). On the surface, grooves borders were very clean, without visible thermal effects. Stromal organization did not show any visible disturbance around the cut.

YZ sections (A.3 and B.3) showed irregular groove's depth due to corneal curve. Grooves, initially on the stroma surface, are then buried in the tissue. In this case, grooves depths were no longer measured.

XZ sections (A.2 and B.2) showed typical images used to grooves depth measurements.

Figure 3 showed AR for different configurations of fluence/pulse and pulses number. In order to determine groove depth and AR on stromal surface, averaged values on several pictures in the $\mathrm{X}-\mathrm{Y}$ plan were deduced to minimize measurement errors. Each AR corresponded to an average of ten measurements.

On figure 3.A and 3.B we can saw an increase in AR with total mean fluence for a constant pulse recovery and a variable fluence/pulse. On the other hand, figure 3.C and 3.D showed a decrease in AR with total mean fluence, but for a constant fluence/pulse and a variable pulses number. These curves showed that for a same total mean fluence $\mathrm{AR}$ can be very different, depending on fluence/pulse and pulses number. An increase in fluence/pulse seemed to augment the AR, while an increase in pulses number seemed to reduce it.

Anterior and posterior stromal ARs were quite high, in the $\mu \mathrm{m} /$ pulse range. On the four curves, results showed significantly higher ARs of a mean $23 \pm 12 \%( \pm \mathrm{SD})$; $(\mathrm{P}=0.0038)$ in posterior corneal stroma than in anterior stroma.

These observations were compatible with other studies realized on corneal stroma, showing higher interwoven lamellar organization and higher shear moduli in anterior than in posterior stroma [9-11]. However, in a practical point of view, this slight difference in AR could be easily compensate by modifying cutting parameters, and did not explain difficulties usually encountered to cut posterior stroma with FL. Optical scattering induced by the laser beam passes through the stroma layers, already identified as a limiting factor [7], was the most probable cause of these difficulties. 

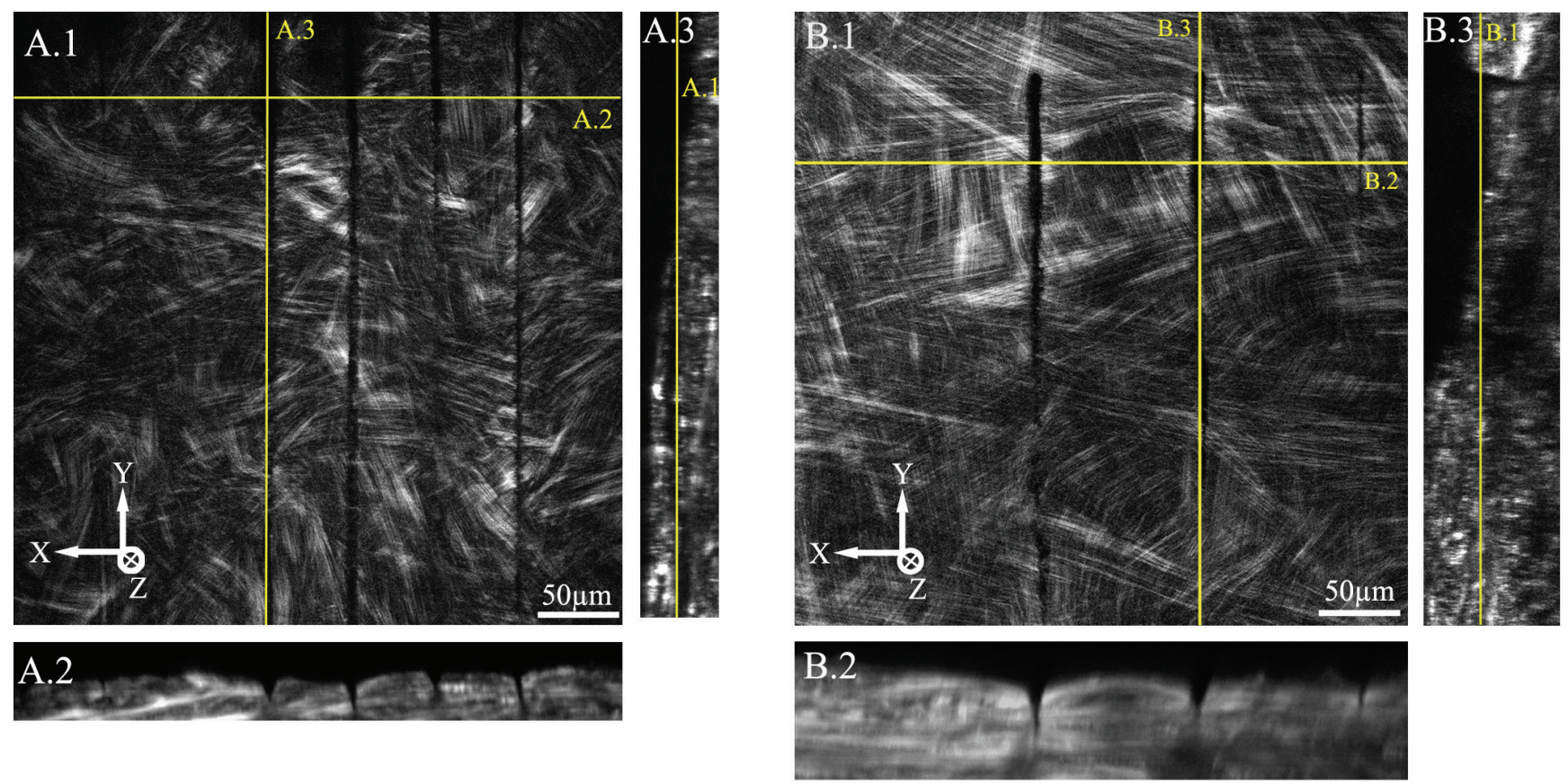

Figure 2: SHG imaging microscopy of grooves in corneal stroma. (A) anterior stroma, (A.1) XY section, grooves parameters (left to right) : $1.1 \mathrm{~mW}$, 11.5pulses ; 3.4mW, 2.3pulses; 3.4mW, 11.5pulses; 3.4mW, 2.3pulses; 3.4mW, 11.5pulses, (A.2) XZ section, mean on several images (A.3) YZ section, mean on several images. (B) Posterior stroma, (B.1) XY section, grooves parameters (left to right) : $3.4 \mathrm{~mW}, 11.5$ pulses; $3.4 \mathrm{~mW}, 2.3 \mathrm{pulses} ; 1.1 \mathrm{~mW}$, 11.5pulses, (B.2) XZ section, mean on several images, (B.3) YZ section, mean on several images.
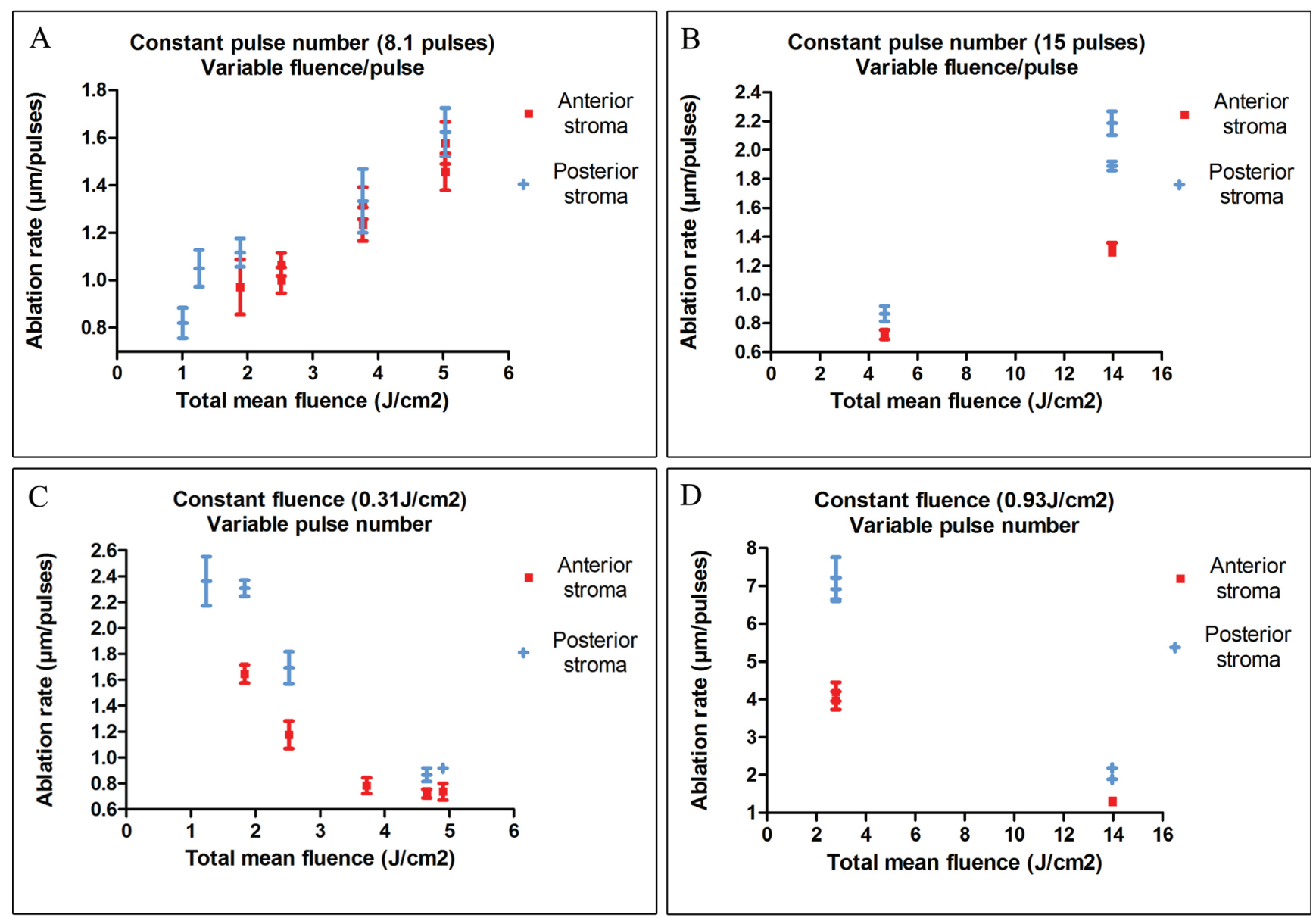

Figure 3 : Ablation rate versus total mean fluence. (A) constant pulse number (8.1pulses) and variable fluence/pulse; (B) constant pulse number (15 pulses) and variable fluence/pulse; (C) constant fluence/pulse $(0.31 \mathrm{~J} / \mathrm{cm} 2)$ and variable pulse number; (D) constant fluence/pulse $(0.93 \mathrm{~J} / \mathrm{cm} 2)$ and variable pulse number. The error bars represent the $95 \%$ Confidence Interval. 


\section{Conclusion}

A systematic study on AR on anterior and posterior surface of human corneal stroma is reported. Second harmonic generation imaging of ablated stroma has been used to determine AR. Results show that femtosecond cut and groove's depth control are executable on the surface of corneal stroma, a very inhomogeneous substrate. Stromal $\mathrm{AR}$ is quite high, in the $\mu \mathrm{m} /$ pulse order. For a constant total mean fluence, AR is very dependent of process parameters (fluence/pulse and pulses recovery). ARs were significantly higher in posterior corneal stroma on a mean $23 \pm 12 \%$ $( \pm$ SD). Limitations encountered to realize deep corneal cut can therefore not be explained by the difference in collagen organization but by the optical scattering during stromal crossing.

\section{Acknowledgments}

(1) The authors thank the staff of the Saint Etienne Eye Banks for their contributions to the study.

\section{References}

[1] K. Koenig, O. Krauss, I. Riemann, Opt. Express, 10 (2002) 171-176.

[2] M. Han, L. Zickler, G. Giese, M. Walter, F.H. Loesel, J.F. Bille, J. Biomed. Opt., 9 (2004) 760-766.

[3] K.R. Sletten, K.G. Yen, S. Sayegh, F. Loesel, C. Eckhoff, C. Horvath, M. Meunier, T. Juhasz, R.M. Kurtz, Ophthalmic Surgery and Lasers, 30 (1999) 742-749.

[4] H.K. Soong, S. Mian, O. Abbasi, T. Juhasz, Ophthalmology, 112 (2005) 44-49.
[5] A. Ertan, J. Colin, Journal of cataract and refractive surgery, 33 (2007) 1303-1314.

[6] Z. Nagy, A. Takacs, T. Filkorn, M. Sarayba, Journal of refractive surgery (Thorofare, N.J.: 1995), 25 (2009) 1053-1060.

[7] V. Nuzzo, M.1. Savoldelli, J.-M. Legeais, K. Plamann, J. Biomed. Opt., 15 (2010) 038003.

[8] S. Sikder, R.W. Snyder, Cornea, 25 (2006) 416-422.

[9] M. Winkler, D. Chai, S. Kriling, C.J. Nien, D.J. Brown, B. Jester, T. Juhasz, J.V. Jester, Invest Ophthalmol Vis Sci, 52 (2011) 8818-8827.

[10] N. Morishige, W.M. Petroll, T. Nishida, M.C. Kenney, J.V. Jester, J Cataract Refract Surg, 32 (2006) 1784-1791.

[11] S.J. Petsche, D. Chernyak, J. Martiz, M.E. Levenston, P.M. Pinsky, Invest Ophthalmol Vis Sci, 53 (2012) 873-880.

[12] R.M. Kurtz, X. Liu, V.M. Elner, J.A. Squier, D. Du, G.A. Mourou, Journal of Refractive Surgery (Thorofare, N.J.: 1995), 13 (1997) 653-658.

[13] D. Giguère, G. Olivié, F.o. Vidal, S.p. Toetsch, G. Girard, T. Ozaki, J.-C. Kieffer, O. Nada, I. Brunette, J. Opt. Soc. Am. A, 24 (2007) 1562-1568.

[14] G. Olivié, D. Giguère, F. Vidal, T. Ozaki, J.-C. Kieffer, O. Nada, I. Brunette, Opt. Express, 16 (2008) 4121-4129.

[15] H. Sun, M. Han, M.H. Niemz, J.F. Bille, Lasers in Surgery and Medicine, 39 (2007) 654-658.

(Received: June 12, 2012, Accepted: October 24, 2012) 